\title{
A Closed-loop Readout Configuration for Mode-localized Resonant MEMS Sensors
}

\author{
Chun Zhao, Milind Pandit, Student Member, IEEE, \\ Boqian Sun, Guillermo Sobreviela, Xudong Zou, Member, IEEE, Ashwin Seshia, Senior Member, IEEE,
}

\begin{abstract}
This paper presents the first experimental results on the closed-loop characterization of a mode-localized microelectromechanical resonator system. Comparisons between the closed-loop oscillator approach and the open-loop frequency sweep approach show good agreement of output metrics including amplitude ratios and mode frequencies. This new approach enables real-time measurements using emerging mode-localized resonant sensors and represents an important step towards realizing sensors based on this measurement principle.
\end{abstract}

Index Terms-Mode localized resonant sensor, self-sustained oscillator

\section{INTRODUCTION}

$\mathbf{M}$ ODE-localized resonant MEMS sensors utilize the principle of vibration mode localization in weakly coupled resonator arrays to transduce variations in structural parameters as shifts in mode shapes [1]. This technique has been applied to devices such as gravimetric sensors [2], force sensors [3], displacement sensing [4] and accelerometers [5]. Mode-localized resonant sensors have been shown to provide distinctive advantages over frequency shift based resonant sensors including significantly enhanced sensitivity (between two to four orders of magnitude) [1]-[5] and passive immunity to environmental effects [2], [6].

However, previous predominant characterization of modelocalized sensors has been based on open-loop read-out techniques, where the modal amplitudes are recorded using swept frequency measurements on a network analyzer limiting measurement bandwidth and scalability for practical applications. As opposed to the open-loop measurement approach, a closedloop approach utilizing a self-sustaining oscillation configuration facilitates automatically tracking of the resonance mode of interest for timing applications [7]-[9] and single-resonator sensors [10]-[12]. In spite of oscillators having been realized based on coupled resonator arrays for timing applications [13], a suitable oscillator configuration has not yet been developed and applied to mode-localized sensors. This is one of the major issues that hinders this progress of the emerging sensing technology [14].

In this paper, a self-sustaining oscillation loop for a $2 \mathrm{DoF}$ mode-localized resonant sensor is demonstrated; closed-loop characterization of the mode-localized sensors, as well as realtime monitoring of the mode-localization effect, is presented for the first time. Closed-loop characterization results of the prototype sensor are compared with open-loop measurements, and a good agreement between both approaches can be observed. This demonstrates the validity of the closedloop configuration proposed here. In addition, it has been

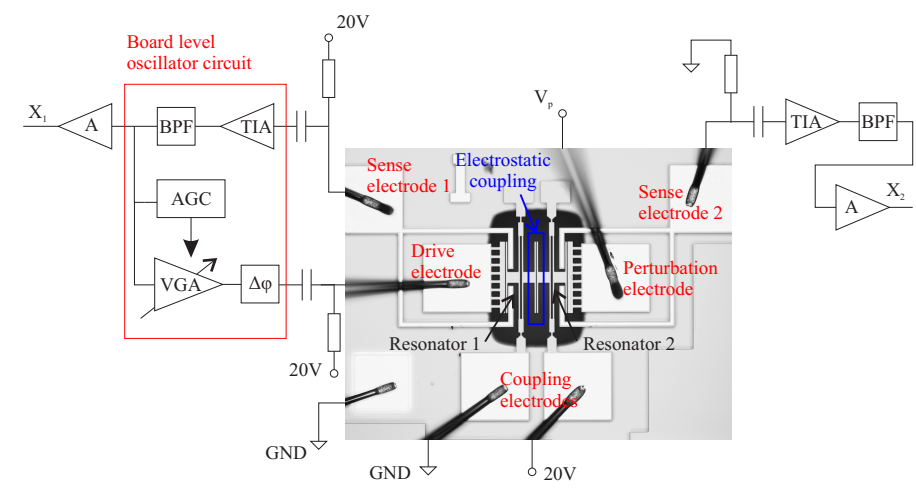

Fig. 1. Oscillator schematics including the optical micro-graph of the prototype mode-localized resonant sensor.

shown that this new read-out technique also maintains the enhanced sensitivity of the mode-localized sensor relative to the approach based on tracking resonant frequency shifts.

\section{MEMS RESONATOR DESIGN}

The frequency determining element in the oscillator loop is a pair of 2DoF electrostatically coupled MEMS resonators, fabricated using the commercially available SOIMUMPS process (MEMSCAP Inc.). The resonating structures are shown in Fig. 1. This design is similar to that of previously reported structures [1]. The structural dimensions of both DETF resonators are identical with $300 \mu \mathrm{m}$ in length $(L), 6 \mu \mathrm{m}$ in width $(w)$ and $25 \mu \mathrm{m}$ in thickness $(t)$, yielding a primary mode resonant frequency of $249.5 \mathrm{kHz}$. The electrostatic coupling strength is defined by a motion dependent capacitance with an original gap of $d=2 \mu \mathrm{m}$ and an overlapping area of $A=260 \mu \mathrm{m} \times 25 \mu \mathrm{m}$. With a DC potential difference of $20 \mathrm{~V}$, the coupling strength normalized to the effective stiffness of the resonator can be estimated as $\kappa=K_{c} / K=2.66 \times 10^{-3}$, indicating a weak coupling for highly sensitive mode-localized sensors. It should be pointed out that the coupling can be even lower to enhance the sensitivity even further. However, in a mode localized resonant sensor, design trade-offs exist between sensitivity, dynamic range and linearity [15]. Moreover, decreasing the coupling voltage also increases the motional resistance. In order to maintain the same motional resistance to facilitate the oscillator design, configurations of the DC bias voltages on driving and sensing electrodes would become complicated and therefore not suitable for practical sensor implementation. 
The weakly coupled resonator system has two vibrational modes due to the weak coupling. Both resonators are designed to be balanced in an ideal situation, where for operation in either mode, the amplitudes of both resonators are identical. When there is a symmetry-breaking perturbation introduced into the system, the previously evenly distributed modal energy is now localized, and the balance in amplitudes is also disrupted. In this case, the degree of mode localization can be measured by the amplitude ratio as a readout metric of the perturbation [15]. In the case of stiffness perturbation $\Delta K / K$, this relationship is given by [5]:

$$
\mid \text { Amplitude Ratio }|=| \frac{\left(\frac{1}{\kappa} \frac{\Delta K}{K}\right) \pm \sqrt{\left(\frac{1}{\kappa} \frac{\Delta K}{K}\right)^{2}+4}}{2} \mid
$$

where $\kappa=K_{c} / K$ is the predefined normalized coupling strength. It is worth mentioning that for the prototype sensor, an external perturbation voltage $V_{p}$ is applied to simulate a stiffness perturbation. Given the permittivity of vacuum $\varepsilon_{0}$ and bias voltage $V_{d c}=20 \mathrm{~V}$, the electrostatic stiffness perturbation can be expressed as:

$$
\Delta K / K=-\frac{\varepsilon_{0} A\left[\left(V_{p}-V_{d c}\right)^{2}-V_{d c}^{2}\right]}{32 d^{3} t}\left(\frac{w}{L}\right)^{3}
$$

where $w, L, t, d$ and $A$ are the predefined parameters of the device: width, length, thickness, air gap and overlapping area, respectively. $V_{p}$ denotes the perturbation voltage. The calculated $\Delta K / K$ ranges from $-3.3 \times 10^{-3}$ to $2 \times 10^{-3}$ as the perturbation voltage changes from $-10 \mathrm{~V}$ to $10 \mathrm{~V}$. This is in the same order as the coupling stiffness strength.

The device is then placed in the vacuum chamber with an ambient pressure of approximately 10mTorr. Open-loop electrical transmission measurements are conducted and a Qfactor of 17000 is obtained.

\section{OSCILLATOR DESIGN}

The oscillators are based on a sine-wave output oscillator topology including an automatic gain control (AGC) scheme to ensure that the resonators are suitably biased in the linear regime [16]. Before the implementation of the oscillator, an optimal strategy for actuating the resonant device should be determined based on the frequency response characterization of the device. The frequency response of the device is measured using an open-loop technique with a network analyzer (4396B, Agilent), and is shown in Fig. 2, for a perturbation voltage range from $-10 \mathrm{~V}$ to $10 \mathrm{~V}$. When the actuation voltage was $22 \mathrm{mVrms}$, the linear response is shown in Fig. 2a. Also shown in the figure Fig. $2 b$ is the nonlinear responses when the resonator is driven with higher amplitudes of $31 \mathrm{mVrms}$ and $39 \mathrm{mV} r m s$. It can be seen that bifurcation occurs when driven with $39 \mathrm{mVrms}$. From the unbalanced response of the magnitude of resonator 1 , it can be observed that the sensor is already biased in a strongly localized region due to fabrication tolerances, using the theory previously reported [3], [5]. This is one of the desired operating regimes for the mode-localized sensor, due to enhanced sensitivity and linear response [15]. Furthermore, for oscillator design, this
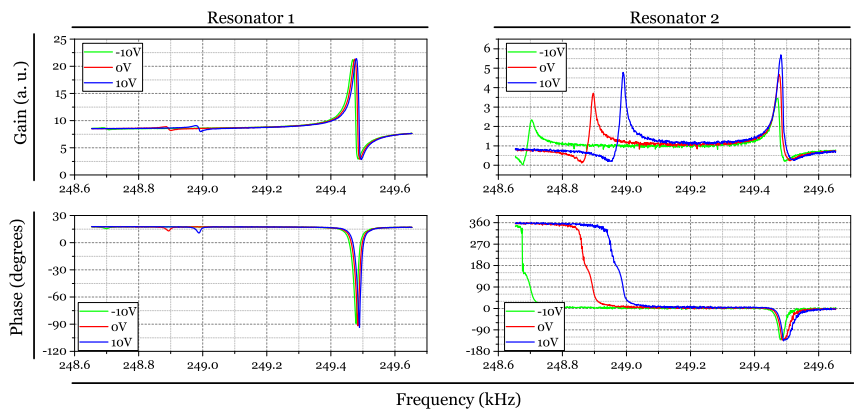

(a) Open loop measurement using $22 \mathrm{mV} r m s$ in the linear regime

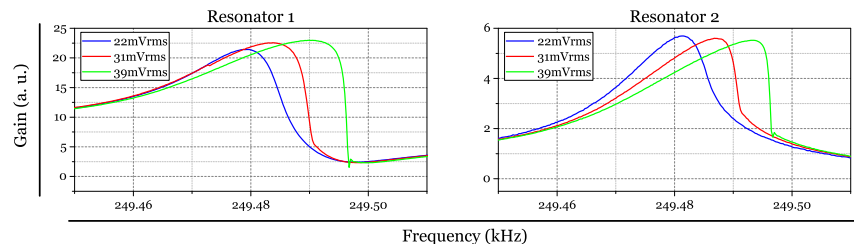

(b) Open loop measurement using different drive voltage, $V_{p}=10 \mathrm{~V}$

Fig. 2. (a) Open-loop measurement results of the prototype mode-localized resonant sensor within the supposed working range of $-10 \mathrm{~V} \leq V_{p} \leq 10 \mathrm{~V}$, with a drive voltage of $22 \mathrm{mV}_{r m s}$, showing both modes for both resonators. The frequency span is $1 \mathrm{kHz}, 801$ points and $1.25 \mathrm{~Hz}$ frequency steps. (b) Open-loop measurement results of the second mode, which shows highest amplitudes at $V_{p}=10 \mathrm{~V}$, with three different actuation voltages, showing that the resonators show nonlinear behavior with bifurcation with an actuation voltage of $39 \mathrm{mV}_{\text {rms }}$.

makes the circuit design simpler. First of all, at the second mode, resonator 1 has constant amplitudes across the supposed working range, as opposed to varying amplitudes of resonator 2. This property facilitates the design of the variable gain amplifier, so that further calibration of the feedback gain is not required. On the contrary, if resonator 2 is used to maintain the drive voltage constant, potential issues, such as resonator 1 could be driven into the nonlinear domain, or the oscillator gain being insufficient to maintain the oscillation, could arise if the feedback gain is not tuned properly during operation. Secondly, the oscillator locks on the second mode only as the magnitude response is more than $10 \mathrm{~dB}$ higher than that of the first mode. It should be noted that the initial imbalance in the system, which brought the device to the desired operating region, was due to fabrication tolerances. However, in general cases, the mode-localized sensor can be engineered to operate in this described region through additional tuning utilizing an external DC voltage [3]. The required stiffness perturbation to tune an ideal balanced mode-localized resonant sensor into the desired region (i.e. minimum amplitude ratio of 3.8, equivalent to that of $V_{p}=10 \mathrm{~V}$ in this study), can be calculated as $\Delta K / K=-1 \times 10^{-2}$, based on Eq. 1. Substituting $V_{p}$ in Eq. 2, with a tuning voltage, $V_{t}$, it can be estimated that a tuning voltage of $63.9 \mathrm{~V}$ or $-23.9 \mathrm{~V}$ is required. This voltage can be potentially reduced by decreasing the coupling voltage. Therefore, resonator 1 was used to generate and maintain the actuation voltage to excite the resonators at the second mode.

The self-sustained oscillator circuit consists of a transimpedance amplifier (TIA) (ADA4637, Analog Devices Inc), a bandpass filter (BPF) (ADA4897, Analog Devices Inc), an automatic gain control (AGC) stage (precision peak detector), 


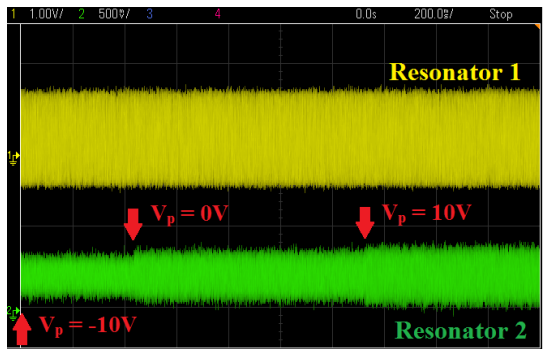

(a)

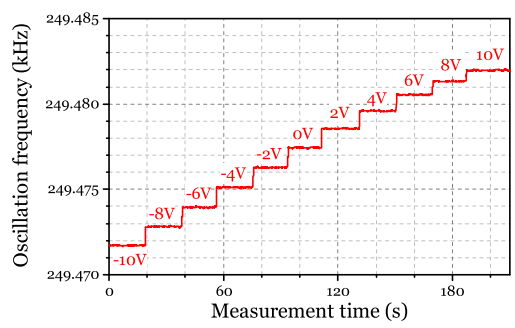

(b)

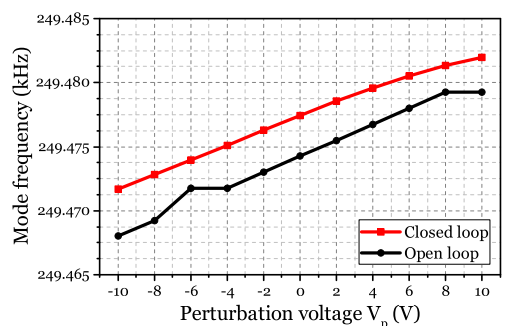

(c)

Fig. 3. Real-time monitoring of the mode localization effect as well as the frequency shift. Coarse real-time amplitude traces are shown in (a), illustrating the approximate trend of amplitude variations in real-time, with detailed steady-state response shown in Fig. 4. Real-time oscillation frequencies with respect to external perturbation voltages are shown in (b) and are compared to open-loop frequencies in (c). It should be noted that the open-loop frequencies can have a frequency step error of $\pm 1.25 \mathrm{~Hz}$ in the experiment. The kinks in the open-loop curve at $-6 \mathrm{~V}$ and $10 \mathrm{~V}$ are likely due to this error.
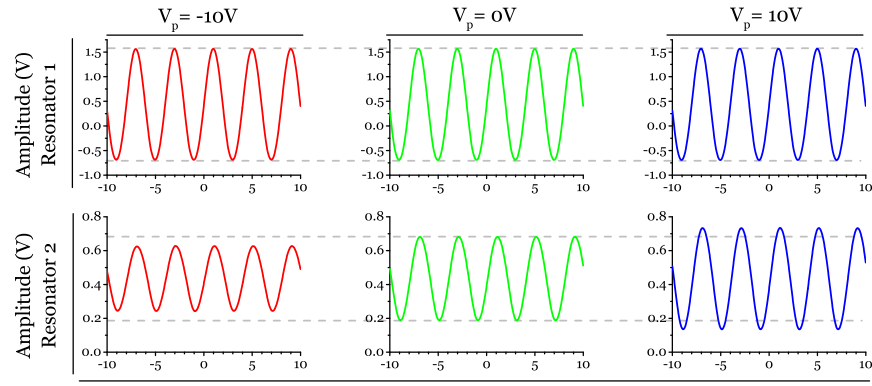

Time $(\mu \mathrm{s})$

Fig. 4. Measured steady-state time domain response of the mode-localized resonant device in a closed-loop configuration with $V_{p}=-10,0$ and $10 \mathrm{~V}$, with a drive voltage of $21.2 \mathrm{mV}_{r m s}$. The amplitude of resonator 1 was maintained thanks to the AGC circuit, while the amplitude response of resonator 2 was varied corresponding to the external perturbation, demonstrating the mode localization effect.

a variable gain amplifier (VGA) (AD600, Analog Devices Inc), a phase shifter $(\Delta \phi)($ AD8045) and a voltage divider (not shown). The TIA has a transimpedance gain of $1 \mathrm{M} \Omega$ and a simulated bandwidth of $760 \mathrm{kHz}$, which is sufficient for the resonant frequency of approximately $250 \mathrm{kHz}$. The BPF is a second-order filter with a central frequency of $250 \mathrm{kHz}$ and a $3-\mathrm{dB}$ bandwidth of $50 \mathrm{kHz}$. Higher order filters could be used to improve the noise, but would also increase the risk of the loop oscillating even in the absent of the MEMS resonator. The AGC stage regulates the sinusoidal signal and filters out the high frequency components, leaving a DC voltage that monotonically increases as the signal level increases, and vice versa. This DC voltage is then used in the VGA to control the feedback gain in the feedback loop. It should be pointed out that a further amplification stage is placed in the readout path, to increase the output signal level. The amplitude of resonators 1 and 2 are measured using an oscilloscope (DSOX2014, Keysight Technologies); the mode frequencies are measured using a frequency counter (53230A, Keysight Technologies).

\section{EXPERIMENTAL RESULTS}

After calibrating the loop phase, the oscillator was then tested in the closed-loop configuration. The rms amplitude of the drive signal was measured using a 6.5-digit digital multimeter (34410A, Keysight Technologies), and was found to be $21.2 \mathrm{mV}_{r m s}$, which is lower than the drive voltage of $22 \mathrm{mV}_{r m s}$ used for linear open-loop characterizations. During the entire perturbation test, the drive voltage was monitored; without further effort of calibration, the reading of the drive voltage was observed to be smaller than $22 \mathrm{mV}_{r m s}$ throughout. This ensured that both resonators were operating in the linear regime.

We demonstrate the capability of monitoring the mode localization effect first. The amplitudes of both resonators 1 and 2 were measured coarsely simultaneously using an oscilloscope, while the perturbation voltage was varied from $-10 \mathrm{~V}$ to $0 \mathrm{~V}$ then to $10 \mathrm{~V}$. The real-time measurements are displayed in Fig. 3a. It can be clearly seen that the amplitude of resonator 1 remained constant throughout the test, while the amplitude of resonator 2 was increased in steps as the $V_{p}$ was changed from $-10 \mathrm{~V}$ to $0 \mathrm{~V}$ and then to $10 \mathrm{~V}$. In a similar manner, the oscillation frequency was also measured using a frequency counter, while the perturbation voltage $V_{p}$ was altered from $-10 \mathrm{~V}$ to $10 \mathrm{~V}$ in $1 \mathrm{~V}$ steps. This tracking in oscillation frequency with response to the external perturbation is demonstrated in Fig. 3b. Using the mean frequency values obtained in this way, the closed-loop oscillation frequency are plotted versus the perturbation voltage $V_{p}$. The comparison to the second mode frequency measured using an open-loop approach is shown in Fig. 3c. It can be seen that the oscillation frequency in a closed-loop configuration matched well with the open-loop oscillation frequency. The discrepancies of less than $4 \mathrm{~Hz}$ can be explained by the frequency drifts over time between the open-loop and closed-loop measurements. It could also be attributed to the frequency offset caused by a phase shift due to different loading conditions between open-loop calibration and the closed-loop configuration.

We then characterized the mode-localized sensor output as reported previously [3], by measuring the amplitude ratio $\left(\left|X_{1}\right| /\left|X_{2}\right|\right)$. The amplitudes were measured using an oscilloscope over 2048 averages to obtain more accurate readings; the averaged steady-state responses are shown in Fig. 4. The calculated variations of amplitude ratios are plotted against the calculated equivalent normalized stiffness perturbation $(\Delta K / K)$ in Fig. 5. It can be observed that the closed-loop response agreed well with the open-loop measurements. An explanation of the discrepancies at larger amplitude ratios 


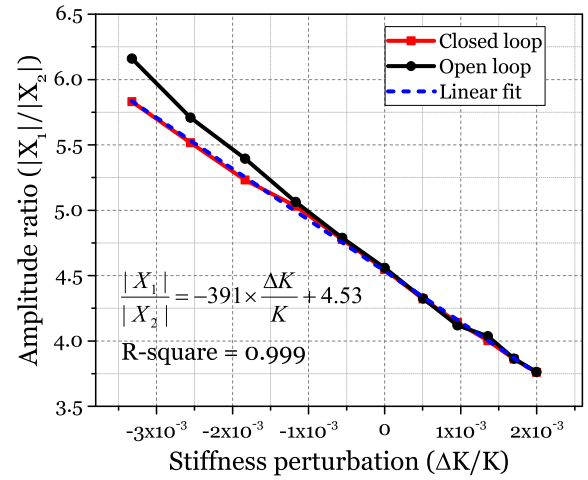

Fig. 5. Amplitude ratios variations for varying the normalized stiffness perturbations $(\Delta K / K)$. The amplitude of resonator 2 is reduced with a negative increasing stiffness perturbation, which becomes more sensitive to a phase error off the resonance, in presence of feedthrough current. Therefore, the discrepancies at higher stiffness perturbations could be attributed to a phase error in closing the loop.

can be the offset away from the resonance frequency. As the amplitude of resonator 2 decreases, the feedthough effect becomes significant, thus an offset away from the resonance frequency can lead to larger amplitude changes. The fitted linear sensitivity using amplitude ratio as an output metric showed a sensitivity of $\left(\frac{\left|X_{1}\right| /\left|X_{2}\right|}{\Delta K / K}\right)=-391$. Using the conventional figure-of-merit for comparison [1], [2], compared to the single resonant sensor with an estimated sensitivity of $\left(\frac{\Delta f / f_{0}}{\Delta K / K}\right)=0.5$, an improvement of 782 times in fractional parametric sensitivity is estimated. Thus, using the closed-loop configuration, the improvements in parametric sensitivity using the mode-localized resonant sensor is successfully maintained.

\section{CONCLUSION}

In this letter, we have reported a closed-loop read-out scheme for mode-localized resonant sensors, which represents an important step forward towards enabling practical realization of such devices. This new approach enables realtime monitoring of the amplitude responses of the coupled resonators at a desired mode while maintaining the improved sensitivity. Future work should include the bandwidth characterization of the mode-localized sensor utilizing the closedloop approach. In addition, the resolution of amplitude detection is inferior to the that of frequency detection. Therefore, one of the key priorities for future study should focus on improving the amplitude measurement resolution for this particular sensor. This in conjunction with further developments to address improvements in response time will enable the practical realization of fully integrated sensor systems.

\section{ACKNOWLEDGMENT}

Funding from Innovate UK and the Natural Environment Research Council is gratefully acknowledged.

\section{REFERENCES}

[1] Pradyumna Thiruvenkatanathan, Jize Yan, Jim Woodhouse, and Ashwin A Seshia. Enhancing parametric sensitivity in electrically coupled mems resonators. Journal of Microelectromechanical Systems, 18(5):1077-1086, 2009.

[2] Matthew Spletzer, Arvind Raman, Alexander Q Wu, Xianfan Xu, and Ron Reifenberger. Ultrasensitive mass sensing using mode localization in coupled microcantilevers. Applied Physics Letters, 88(25):254102, 2006.

[3] Chun Zhao, Graham S Wood, Jianbing Xie, Honglong Chang, Suan Hui $\mathrm{Pu}$, and Michael Kraft. A force sensor based on three weakly coupled resonators with ultrahigh sensitivity. Sensors and Actuators A: Physical, 232:151-162, 2015.

[4] P Thiruvenkatanathan and AA Seshia. Mode-localized displacement sensing. Journal of Microelectromechanical Systems, 21(5):1016-1018, 2012.

[5] Hemin Zhang, Boyang Li, Weizheng Yuan, Michael Kraft, and Honglong Chang. An acceleration sensing method based on the mode localization of weakly coupled resonators. Journal of Microelectromechanical Systems, 25(2):286-296, 2016.

[6] Pradyumna Thiruvenkatanathan, Jize Yan, and Ashwin A Seshia. Differential amplification of structural perturbations in weakly coupled mems resonators. IEEE transactions on ultrasonics, ferroelectrics, and frequency control, 57(3):690-697, 2010.

[7] Clark T-C Nguyen. Mems technology for timing and frequency control. In Proceedings of the 2005 IEEE International Frequency Control Symposium and Exposition, 2005., pages 11-pp. IEEE, 2005.

[8] James C Salvia, Renata Melamud, Saurabh A Chandorkar, Scott F Lord, and Thomas W Kenny. Real-time temperature compensation of mems oscillators using an integrated micro-oven and a phase-locked loop. Journal of Microelectromechanical Systems, 19(1):192-201, 2010.

[9] Roozbeh Tabrizian, Mauricio Pardo, and Farrokh Ayazi. A $27 \mathrm{mhz}$ temperature compensated mems oscillator with sub-ppm instability. In Micro Electro Mechanical Systems (MEMS), 2012 IEEE 25th International Conference on, pages 23-26. IEEE, 2012.

[10] M Li, EJ Ng, VA Hong, CH Ahn, Y Yang, TW Kenny, and DA Horsley. Lorentz force magnetometer using a micromechanical oscillator. Applied Physics Letters, 103(17):173504, 2013.

[11] Xudong Zou, Pradyumna Thiruvenkatanathan, and Ashwin A Seshia. A seismic-grade resonant mems accelerometer. Journal of Microelectromechanical Systems, 23(4):768-770, 2014.

[12] Giacomo Langfelder, Alessandro Caspani, and Alessandro Tocchio. Design criteria of low-power oscillators for consumer-grade mems resonant sensors. IEEE Transactions on Industrial Electronics, 61(1):567-574, 2014.

[13] Seungbae Lee and CT-C Nguyen. Mechanically-coupled micromechanical resonator arrays for improved phase noise. In Frequency Control Symposium and Exposition, 2004. Proceedings of the 2004 IEEE International, pages 144-150. IEEE, 2004.

[14] Chun Zhao, Mohammad H Montaseri, Graham S Wood, Suan Hui Pu, Ashwin A Seshia, and Michael Kraft. A review on coupled mems resonators for sensing applications utilizing mode localization. Sensors and Actuators A: Physical, 249:93-111, 2016.

[15] Chun Zhao, Graham S Wood, Jianbing Xie, Honglong Chang, Suan Hui $\mathrm{Pu}$, and Michael Kraft. A three degree-of-freedom weakly coupled resonator sensor with enhanced stiffness sensitivity. Journal of Microelectromechanical Systems, 25(1):38-51, 2016.

[16] Lin He, Yong Ping Xu, and Moorthi Palaniapan. A cmos readout circuit for soi resonant accelerometer with 4-bias stability and 20-resolution. IEEE journal of solid-state circuits, 43(6):1480-1490, 2008. 